\title{
Simplicity of Jordan Superalgebras and Relations with Lie Structures
}

\author{
CONSUELO MARTÍNEZ
}

\section{Generalities}

Superalgebras appeared (as graded algebras) in the context of algebraic topology and homological algebra, but they endured a new impetuous development due to Physics and the attempt to capture "the supersymmetry" between Bosons and Fermions. Later, superalgebras proved to be important as purely algebraic objects, they have produced new ideas and methods and have helped to solve some old algebraic problems.

As in any algebraic structure theory, the primary problem is the classification of simple structures. This paper is an attempt of a survey of what is known about simplicity of (associative, Lie and Jordan) superalgebras.

In what follows algebra will mean algebra over a field $F$.

Definition 1.1. A superalgebra $A=A_{\overline{0}}+A_{\overline{1}}$ is a $\mathbb{Z}_{2}$-graded algebra, that is, $A_{\bar{i}} \cdot A_{\bar{j}} \subseteq A_{i \overline{+} j}$. So $A_{\overline{0}}$ is the even part of $A$ and is a subalgebra and $A_{\overline{1}}$ is the odd part of $A$ and is a bimodule over $A_{\overline{0}}$.

For instance, if $V$ is a vector space of countable dimension, and $G(V)=G(V)_{\overline{0}}+G(V)_{\overline{1}}$ is the Grassmann algebra over $V$, that is, the quotient of the tensor algebra over the ideal generated by the symmetric tensors, then $G(V)$ is a superalgebra. Its even part is the linear span of all products of even length and the odd part is the linear span of all products of odd length.

If $A$ is a superalgebra, its Grassmann enveloping algebra is the subalgebra of $A \otimes G(V)$ given by $G(A)=A_{\overline{0}} \otimes G(V)_{\overline{0}}+A_{\overline{1}} \otimes G(V)_{\overline{1}}$

By using the Grassmann enveloping algebra it is easy to define the notion of $\mathcal{V}$-superalgebra, where $\mathcal{V}$ is a variety of algebras.

Partially supported by PB97-1291-C03-01. 
Definition 1.2. If $\mathcal{V}$ is a variety of algebras, a superalgebra $A=A_{\overline{0}}+A_{\overline{1}}$ is a $\mathcal{V}$ superalgebra if $G(A) \in \mathcal{V}$.

\section{Examples.}

1. An associative superalgebra is just an associative $\mathbb{Z}_{2}$-graded algebra.

2. An algebra $L$ is a Lie algebra if it satisfies:

L1. Anticommutativity $[a, b]=-[b, a]$,

L2. Jacobi identity $[[a, b], c]+[[b, c], a]+[[c, a], b]=0$.

A superalgebra $L=L_{\overline{0}}+L_{\overline{1}}$ is a Lie superalgebra if it satisfies

SL1. Superanticommutativity $[a, b]=-(-1)^{|a||b|}[b, a]$,

SL2. Super Jacobi identity

$$
[[a, b], c]+(-1)^{|a|(|b|+|c|)}[[b, c], a]+(-1)^{|c|(|a|+|b|)}[[c, a], b]=0,
$$

where $|a|=i$ if $a \in A_{\bar{i}}(i=0,1)$ and $a, b, c \in L_{\overline{0}} \cup L_{\overline{1}}$.

3. A Jordan algebra $J$ is an algebra that satisfies

J1. Commutativity $a \cdot b=b \cdot a$

J2. Jordan identity $a^{2} \cdot(b \cdot a)=\left(a^{2} \cdot b\right) \cdot a$

or equivalently the linearization:

$$
\begin{aligned}
& (a \cdot b) \cdot(c \cdot d)+(a \cdot c) \cdot(b \cdot d)+(a \cdot d) \cdot(b \cdot c)= \\
& ((a \cdot b) \cdot c) \cdot d+((a \cdot d) \cdot c) \cdot b+((b \cdot d) \cdot c) \cdot a .
\end{aligned}
$$

$J=J_{\overline{0}}+J_{\overline{1}}$ is a Jordan superalgebra if it satisfies:

SJ1. Supercommutativity $a \cdot b=(-1)^{|a||b|} b \cdot a$,

SJ2. Super Jordan identity

$$
\begin{gathered}
(a \cdot b) \cdot(c \cdot d)+(-1)^{|b||c|}(a \cdot c) \cdot(b \cdot d)+(-1)^{|b||d|+|c||d|}(a \cdot d) \cdot(b \cdot c)= \\
((a \cdot b) \cdot c) \cdot d+(-1)^{|c||d|+|b| \mid c}((a \cdot d) \cdot c) \cdot b+ \\
(-1)^{|a||b|+|a||c|+|a||d|+|c||d|}((b \cdot d) \cdot c) \cdot a .
\end{gathered}
$$

Remarks. 1. A Lie (resp. Jordan) superalgebra $A=A_{\overline{0}}+A_{\overline{1}}$ is not a Lie (resp. Jordan) algebra (except if $\left.A_{\overline{1}}=(0)\right)$. The even part $A_{\overline{0}}$ is a Lie (resp. Jordan) algebra and $A_{\overline{1}}$ is an $A_{\overline{0}}$-bimodule with an operation $A_{\overline{1}} \times A_{\overline{1}} \longrightarrow A_{\overline{0}}$.

2. The superidentity is obtained from the corresponding identity following the KAPLANSLY RULE: If two homogeneous adjacent variables $a, b$ are exchanged, then the corresponding term is multiplied by $(-1)^{|a||b|}$. 
Let us now consider some examples of superalgebras.

2. Examples of Simple Associative And Lie Superalgebras

\section{Associative Superalgebras.}

(I) $A=M_{m+n}(F), A_{\overline{0}}=\left\{\left(\begin{array}{cc}\star & 0 \\ 0 & \star\end{array}\right)\right\}, A_{\overline{1}}=\left\{\left(\begin{array}{ll}0 & \star \\ \star & 0\end{array}\right)\right\}$.

(II) $A=\left\{\left(\begin{array}{ll}a & b \\ b & a\end{array}\right) \mid a, b \in M_{n}(F)\right\}$.

Theorem 2.1. (C.T.C. Wall, 1963) Every associative simple finite-dimensional superalgebra over an algebraically closed field $F$ is isomorphic to (I) or (II).

\section{Lie Superalgebras.}

1. $s \ell(m, n)$ denotes the Lie superalgebra whose even part is the set of matrices $\left(\begin{array}{ll}\alpha & 0 \\ 0 & \delta\end{array}\right)$, where $\alpha \in M_{m \times m}(F), \delta \in M_{n \times n}(F)$ and $\operatorname{tr} \alpha=\operatorname{tr} \delta$ and the odd part consists of matrices $\left(\begin{array}{ll}0 & \beta \\ \gamma & 0\end{array}\right)$, with $\beta \in M_{m \times n}(F)$ and $\gamma \in M_{n \times m}(F)$.

$$
\begin{aligned}
& \underline{A(m, n)}=s \ell(m+1, n+1) \text { for } m \neq n ; m, n \geq 0 \\
& \left.\underline{A(m, m)}=s \ell(m+1, m+1) /\left\langle I_{2 m+2}\right\rangle ; m\right\rangle 0
\end{aligned}
$$

2. Let $\operatorname{osp}(m, n)$ (orthogonal symplectic superalgebra) be the superalgebra that consists of $m \times n$ matrices, $n=2 r$, of the form:

$$
\left(\begin{array}{ccccc}
a & b & u & x & x_{1} \\
c & -a^{T} & v & y & y_{1} \\
-v^{T} & -u^{T} & 0 & z & z_{1} \\
y_{1}^{T} & x_{1}^{T} & z_{1}^{T} & d & e \\
-y^{T} & -x^{T} & -z^{T} & f & -d^{T}
\end{array}\right)
$$

if $m=2 l+1$, where $a, b, c \in M_{l \times l}(F), b=-b^{T}, c=-c^{T}, u, v \in$ $M_{l \times 1}(F), d, e, f \in M_{r \times r}(F), e=e^{T}, f=f^{T}, x, y, x_{1}, y_{1} \in M_{l \times r}(F)$, $z, z_{1} \in M_{1 \times r}(K)$, and

$$
\left(\begin{array}{cccc}
a & b & x & x_{1} \\
c & -a^{T} & y & y_{1} \\
y_{1}^{T} & x_{1}^{T} & d & e \\
-y^{T} & -x^{T} & f & -d^{T}
\end{array}\right)
$$


if $m=2 l$.

So, the following superalgebras can be considered:

$$
\begin{gathered}
B(m, n)=\operatorname{osp}(2 m+1,2 n), m \geq 0, n>0, \\
D(m, n)=\operatorname{osp}(2 m, 2 n), m \geq 2, n>0, \\
C(n)=\operatorname{osp}(2,2 n-2), n \geq 2 .
\end{gathered}
$$

3. $P(n)$ is the subsuperalgebra of $s \ell(n+1, n+1), n \geq 2$ that consists of matrices $\left(\begin{array}{cc}a & b \\ c & -a^{T}\end{array}\right)$, where $a, b, c \in M_{n+1, n+1}(F), \operatorname{tr} a=0, b=$ $b^{T}, c=-c^{T}$.

4. Let us consider $\tilde{Q}(n)=\left\{\left(\begin{array}{ll}a & b \\ b & a\end{array}\right) \mid \operatorname{tr} b=0\right\}$, a subsuperalgebra of $s \ell(n+1, n+1)$. Its center is $C=\left\langle I_{2 n+2}\right\rangle$. The quotient algebra is a simple superalgebra, $Q(n)=\tilde{Q}(n) / C$.

5. $F(4), G(3), D(2,1, \alpha)$.

Let $A=\left(a_{i j}\right) \in M_{r \times r}(F)$ be a square $r \times r$ matrix and $\tau \subseteq$ $\{1,2, \ldots, r\}$ a subset of the indices set. Then the Contragradient Lie superalgebra $G(A, \tau)$ is the minimal $\mathbb{Z}$-graded Lie superalgebra with local part $\hat{G}(A, \tau)=G_{-1} \oplus G_{0} \oplus G_{1}$ (that is, $G(A, \tau)$ is an epimorphic image of every Lie superalgebra that has $\hat{G}(A, \tau)$ as local part. Its existence has been proved in [6]), where $G_{-1}, G_{0}, G_{1}$ vector spaces of basis $\left\{f_{i}\right\},\left\{h_{i}\right\}$ and $\left\{e_{i}\right\}$, respectively and product given by:

$$
\begin{aligned}
& {\left[e_{i}, f_{j}\right]=\delta_{i j} h_{i}, \quad\left[h_{i}, h_{j}\right]=0,} \\
& {\left[h_{i}, e_{j}\right]=a_{i j} e_{j}, \quad\left[h_{i}, f_{j}\right]=-a_{i} j f_{j},} \\
& \operatorname{deg}_{i}=0, \text { dege }_{i}=\operatorname{deg} f_{i}=0 \text { if } i \notin \tau \text { and } \operatorname{dege}_{i}=\operatorname{deg} f_{i}=1 \text { if }
\end{aligned}
$$
$i \in \tau$.

The Lie superalgebras $D(2,1 ; \alpha), F(4)$ and $G(3)$ are particular cases of Contragradient Lie superalgebras associated to some concrete matrices and with the set $\tau=\{1\}$ in all cases.

$$
\begin{gathered}
D(2,1 ; \alpha)=G(A, \tau), \quad A=D_{\alpha}=\left(\begin{array}{ccc}
0 & 1 & \alpha \\
-1 & 2 & 0 \\
-1 & 0 & 2
\end{array}\right), \\
F(4)=G(A, \tau), \quad A=F_{4}=\left(\begin{array}{cccc}
0 & 1 & 0 & 0 \\
-1 & 2 & -2 & 0 \\
0 & -1 & 2 & -1 \\
0 & 0 & -1 & 2
\end{array}\right),
\end{gathered}
$$




$$
G(3)=G(A, \tau), \quad A=G_{3}=\left(\begin{array}{ccc}
0 & 1 & 0 \\
-1 & 2 & 3 \\
0 & -1 & 2
\end{array}\right) .
$$

Lie superalgebras $A(m, n), B(m, n), C(n), D(m, n), D(2,1, \alpha)$, $F(4), G(3), P(n)$ and $Q(n)$ are called classical superalgebras. They are simple and the action of $L_{\overline{0}}$ on $L_{\overline{1}}$ is completely reducible.

6. Superalgebras of Cartan type $W(n), S(n), \tilde{S}(n), H(n)$.

Let $\Lambda(n)$ denote the Grassmann superalgebra generated by $\xi_{1}, \ldots$, $\xi_{n}$. Then $W(n)=\operatorname{Der} \Lambda(n)$ is the superalgebra of superderivations of $\Lambda(n)$, that is, linear maps that satisfy

$$
D(a b)=D(a) b+(-1)^{|a||D|} a D(b),
$$

where $|D|=0$ if $D\left(L_{\bar{i}}\right) \subseteq L_{\bar{i}}$ and $|D|=1$ if $D\left(L_{\bar{i}}\right) \subseteq L_{\bar{i}+\overline{1}}$. An arbitrary element $D \in W(n)$ can be represented as $D=\sum P_{i} \frac{\partial}{\partial \xi_{i}}$, where $P_{i} \in \Lambda(n)$.

The superalgebra $S(n)$ of $W(n)$ consists of elements $\sum P_{i} \frac{\partial}{\partial \xi_{i}}$ such that $\sum \frac{\partial}{\partial \xi_{i}}\left(P_{i}\right)=0$. That is, $S(n)=\{D \in W(n) \mid D \omega=0\}$, where $\omega$ is a differential form over the associative superalgebra $\Lambda(n), \omega=$ $\theta \xi_{1} \wedge \ldots \wedge \theta \xi_{n}$.

Similarly, $\tilde{S}(n)=\{D \in W(n) \mid D \tilde{\omega}=0\}$, where now $\tilde{\omega}$ is the differential form $\tilde{\omega}=\left(1-\xi_{1} \ldots \xi_{n}\right) \theta \xi_{1} \wedge \ldots \wedge \theta \xi_{n}$, and $n=2 k$.

The subsuperalgebras $S(n)$ and $\tilde{S}(n)$ of $W(n)$ are called special.

Let $\tilde{H}(n)$ denote the subsuperalgebra of $W(n)$ that consists of derivations $\sum P_{i} \frac{\partial}{\partial \xi_{i}}$ such that $D \omega=0$,

$$
\tilde{H}(n)=\{D \in W(n) \mid D \omega=0\},
$$

where $\omega=\left(d \xi_{1}\right)^{2}+\ldots+\left(d \xi_{n}\right)^{2}$.

Then $H(n)=[\tilde{H}(n), \tilde{H}(n)]$ is a subsuperalgebra of $W(n)$ called Hamiltonian.

Theorem 2.2. [3] A simple finite dimensional Lie superalgebra over an algebraically closed field $F$ of characteristic 0 is isomorphic to a simple Lie algebra or to one of the previous $A(m, n), B(m, n)$, $C(n), D(m, n), D(2,1 ; \alpha), F(4), G(3), P(n), Q(n), W(n), S(n)$, $\tilde{S}(n)$, or $H(n)$. 


\section{JORDAN SUPERALGEBRAS}

If $A=A_{\overline{0}}+A_{\overline{1}}$ is an associative superalgebra, we can get a Jordan superalgebra with the same underlying vector space, $A^{(+)}$, by defining the new product $a \cdot b=\frac{1}{2}\left(a b+(-1)^{|a||b|} b a\right)$.

A Jordan superalgebra $J=J_{\overline{0}}+J_{\overline{1}}$ is said to be special if $J \leq$ $A^{(+)}$, for some associative superalgebra $A$. Otherwise, it is called exceptional.

Following Kac's notation (see [7]) we will consider the following Jordan superalgebras:

(A) The superalgebra $A^{(+)}$, where $A=M_{m+n}(F)$ is a full linear superalgebra (see example (I) of simple associative superalgebras).

(Q) The superalgebra $A^{(+)}$, where $A=\left\{\left(\begin{array}{ll}a & b \\ b & a\end{array}\right) \mid a, b \in M_{n}(F)\right\}$ (see example (II) of simple associative superalgebras).

If $A$ is an associative (resp. alternative) superalgebra and $\star: A \rightarrow$ $A$ is an involution: $\left(a^{\star}\right)^{\star}=a,(a b)^{\star}=(-1)^{|a||b|} b^{\star} a^{\star}$, then $H(A, \star)=$ $\left\{a \in A \mid a^{\star}=a\right\}$ is a Jordan subsuperalgebra of $A^{(+)}$(the Jordan superalgebra of "Hermitian elements").

(BC) The associative superalgebra $M_{m+2 n}(F)$ has an involution defined by the matrix $Q=\left(\begin{array}{cc}I_{m} & 0 \\ 0 & S_{2 n}\end{array}\right)$, where

$$
S_{2 n}=\left(\begin{array}{ccccc}
0 & 1 & \cdot & \cdot & \cdot \\
-1 & 0 & \cdot & \cdot & \cdot \\
\cdot & \cdot & \cdot & \cdot & \cdot \\
\cdot & \cdot & \cdot & 0 & 1 \\
\cdot & \cdot & \cdot & -1 & 0
\end{array}\right)
$$

The involution acts in the following way:

$$
\star:\left(\begin{array}{ll}
a & b \\
c & d
\end{array}\right) \rightarrow Q^{-1}\left(\begin{array}{cc}
a^{T} & -c^{T} \\
b^{T} & d^{T}
\end{array}\right) Q, \quad a \in M_{m}(F), \quad d \in M_{2 n}(F) .
$$

The set of Hermitian elements gives the orthosympletic superalgebra $H(A, \star)=\mathbf{o s p}_{\mathbf{m}, \mathbf{2 n}}(\mathbf{F})$. (The same notation as in Lie superalgebras is used.)

(P) Let $A=M_{n+n}(F)$ and consider the (super)involution

$$
\star:\left(\begin{array}{ll}
a & b \\
c & d
\end{array}\right) \longmapsto\left(\begin{array}{cc}
d^{T} & -b^{T} \\
c^{T} & a^{T}
\end{array}\right) .
$$


Then $H(A, \star)=\left\{\left(\begin{array}{cc}a & b \\ c & a^{T}\end{array}\right) \mid a, b, c \in M_{n}(F), b^{T}=-b, c^{T}=c\right\}$ is a simple Jordan superalgebra.

(D) Superalgebra of a superform.

Let $V=V_{\overline{0}}+V_{\overline{1}}$ be a $\mathbb{Z}_{2}$-graded vector space and $\langle\rangle:, V \times V \rightarrow$ $F$ a supersymmetric bilinear form (that is, a bilinear form that is symmetric on the even part $V_{\overline{0}}$, skewsymmetric on $V_{\overline{1}}$ and $\left\langle V_{\overline{0}}, V_{\overline{1}}\right\rangle=$ $(0)=\left\langle V_{\overline{1}}, V_{\overline{0}}\right\rangle$.

Then $J=F 1+V=\left(F 1+V_{\overline{0}}\right)+V_{\overline{1}}$ is a Jordan superalgebra with $J_{\overline{0}}=F 1+V_{\overline{0}}, J_{\overline{1}}=V_{\overline{1}}$, and the product given by $(\alpha 1+v)(\beta 1+w)=$ $(\alpha \beta+\langle v, w\rangle) 1+(\alpha w+\beta v)$.

$\left(\mathbf{D}_{\mathbf{t}}\right)$ Let $J_{t}=\left(F e_{1}+F e_{2}\right)+(F x+F y)$ be the four dimensional superalgebra, $t \neq 0$, with the product given by $e_{i}^{2}=e_{i}, e_{1} e_{2}=0, e_{i} x=$ $\frac{1}{2} x, e_{i} y=\frac{1}{2} y,[x, y]=e_{1}+t e_{2}$. This family of Jordan superalgebras (that depend on the parameter $t$ ) corresponds to the family of 17-dimensional Lie superalgebras $D(2,1, \alpha)$.

(F) The 10-dimensional exceptional Kac superalgebra is the superalgebra whose even part is 6-dimensional, the odd part has dimension 4,

$$
K_{10}=\left[\left(F e_{1}+\sum_{i=1}^{4} F v_{i}\right)+F e_{2}\right]+\left(\sum_{i=1}^{2} F x_{i}+F y_{i}\right)
$$

and the product is given by

$$
\begin{gathered}
e_{i}^{2}=e_{i}, \quad e_{1} e_{2}=0, \quad e_{1} v_{i}=v_{i}, e_{2} v_{i}=0, \quad v_{1} v_{2}=2 e_{1}=v_{3} v_{4} \\
e_{i} x_{j}=\frac{1}{2} x_{j}, \quad e_{i} y_{j}=\frac{1}{2} y_{j}, \quad i, j=1,2 y_{1} v_{1}=x_{2}, \quad y_{2} v_{1}=-x_{1} \\
x_{1} v_{2}=-y_{2}, \quad x_{2} v_{2}=y_{1}, x_{2} v_{3}=x_{1}, \quad y_{1} v_{3}=y_{2}, \\
x_{1} v_{4}=x_{2}, \quad y_{2} v_{4}=y_{1}, \quad\left[x_{i}, y_{i}\right]=e_{1}-3 e_{2}, \\
{\left[x_{1}, x_{2}\right]=v_{1}, \quad\left[y_{1}, y_{2}\right]=v_{2}, \quad\left[x_{1}, y_{2}\right]=v_{3}, \quad\left[x_{2}, y_{1}\right]=v_{4} .}
\end{gathered}
$$

(K) The 3-dimensional Kaplansky superalgebra, $K_{3}=F e+(F x+$ Fy), has the product:

$$
e^{2}=e, \quad e x=\frac{1}{2} x, e y=\frac{1}{2} y, \quad[x, y]=e .
$$

(J) All simple Jordan algebras. 
Theorem 3.1. [3], [6] A simple finite dimensional Jordan superalgebra over an algebraically closed field of zero characteristic is isomorphic to one of the superalgebras $A, B C, D, P, Q, D_{t}, F, K$, or $J$ listed above or to a superalgebra obtained by the Kantor-double process (that will be explained later).

\section{Kantor-Koecher-Tits construction.}

Kac obtained the classification of simple Jordan superalgebras in zero characteristic using the previously known classification of Lie superalgebras and the connection between Lie and Jordan structures via the Kantor-Koecher-Tits process.

Let $L$ be a Lie algebra containing a subalgebra $F e+F h+F f$ which is isomorphic to $s \ell_{2}(F)$, (that is, $[e, f]=h,[f, h]=2 f,[e, h]=-2 e$ ). Suppose that the operator ad $h: L \rightarrow L$ is diagonalizable and the only eigenvalues of ad $h$ are $-2,0,2$. Let us denote $L=L_{-2}+L_{0}+L_{2}$ the decomposition of $L$ into a sum of eigenspaces. Then, following Tits, we can define a structure of a Jordan algebra on $J=L_{-2}$ by: $x_{-2} \cdot y_{-2}=\left[\left[x_{-2}, f\right], y_{-2}\right]$.

The algebra $L$ can be recovered (up to central extensions) from $J$.

Conversely, if $J$ is a Jordan algebra with 1 , there exists a unique (up to isomorphism) pair $s \ell_{2}(F) \subseteq L$ with the above mentioned properties, such that $L_{-2} \simeq J$ and $L$ has zero center. Such Lie algebra is the Kantor-Koecher-Tits construction of $J, K(J)=J^{-}+$ $\left[J^{-}, J^{+}\right]+J^{+}$.

The same can be said about superalgebras. So Kac studied those simple Lie superalgebras that have a short gradation that comes from a "good" $s \ell_{2}(F)$ subalgebra of the even part.

Some Additional Examples in char 3.

In the case of prime characteristic, namely, if char $F=3$, some new simple Jordan superalgebras appear.

$\left(\mathbf{K}_{\mathbf{9}}\right)$ Consider the 10-dimensional Kac superalgebra in characteristic $3, K_{10}=J_{\overline{0}}+J_{\overline{1}}, \quad J_{\overline{0}} \simeq F \oplus B$. Then $K_{9}=B+J_{1}$ is a subsuperalgebra of $K_{10}$.

Let $H_{3}(F)$ denote the set of $3 \times 3$ symmetric matrices over $F$ and let $S_{3}(F)$ be the set of $3 \times 3$ skewsymmetric matrices over $F$. Then we can consider the superalgebra $J$ whose even part is $H_{3}(F)$ and the odd part is a sum of two copies of $S_{3}(F)$, that is, $J_{\overline{0}}=H_{3}(F)$, $J_{\overline{1}}=\overline{S_{3}(F)}+\overline{\overline{S_{3}(F)}}$, with the usual product and the bracket given 
by:

$$
\begin{gathered}
{\left[\overline{S_{3}(F)}, \overline{S_{3}(F)}\right]=\left[\overline{\overline{S_{3}(F)}}, \overline{\overline{S_{3}(F)}}\right]=(0),} \\
{[\bar{a}, \overline{\bar{b}}]=a b+b a, a, b \in S_{3}(F) .}
\end{gathered}
$$

M. Racine proved, with the help of a computer, that $J=J_{\overline{0}}+J_{\overline{1}}$ is a Jordan superalgebra. I. Shestakov found a new realization that proves, in an easier way, that $J$ is a Jordan superalgebra.

I. Shestakov and E. Zelmanov had proved that, if char $F \neq 2,3$, then every prime alternative superalgebra is either associative or isomorphic to a Cayley ring. However I. Shestakov found, in characteristic 3 , two simple alternative superalgebras, denoted $A_{3}$ and $A_{6}$ respectively, that are neither associative nor Cayley rings. Each of them has a natural involution *. The set of Hermitian elements with respect to the involution is in each case a Jordan superalgebra. So $J \simeq H_{3}\left(A_{3}\right)$, while $H_{3}\left(A_{6}\right)$ gives another example of a simple Jordan superalgebra.

Theorem 3.2. [23] Every simple Jordan superalgebra over an algebraically closed field $F$, char $F=p>2$, with its even part semisimple is isomorphic to one of the superalgebras mentioned above.

\section{Jordan Superalgebras Defined by Brackets}

Kantor noticed that a simple Jordan superalgebra was missing in the classification given by Kac in the case of zero characteristic. Such algebra belongs to a general class of superalgebras constructed by Kantor from an associative superalgebra and a Poisson bracket defined over it.

Let $\Gamma=\Gamma_{\overline{0}}+\Gamma_{\overline{1}}$ be an associative commutative superalgebra, $\{\cdot, \cdot\}: \Gamma \times \Gamma \rightarrow \Gamma$ a Poisson bracket, that is, a bilinear map satisfying $\left\{\Gamma_{\bar{i}}, \Gamma_{\bar{j}}\right\} \subseteq \Gamma_{\bar{i}+j}$ and the following two conditions:

P1. $(\Gamma,\{\cdot, \cdot\})$ is a Lie superalgebra,

P2. $\{a b, c\}=a\{b, c\}+(-1)^{|b||c|}\{a, c\} b \quad$ (Leibniz identity)

Let $J=\Gamma+\Gamma x$ be the sum of two copies of $\Gamma$ with the following product defined in $J: a(b x)=(a b) x, \quad(b x) a=(-1)^{|a|}(b a) x$, $(a x)(b x)=(-1)^{|b|}\{a, b\}, \quad J_{\overline{0}}=\Gamma_{\overline{0}}+\Gamma_{\overline{1}} x, J_{\overline{1}}=\Gamma_{\overline{1}}+\Gamma_{\overline{0}} x$.

Theorem 4.1. [7] If $\{\cdot, \cdot\}$ is a Poisson bracket on the associative commutative superalgebra $\Gamma$, then $J=\Gamma+\Gamma x$ is a Jordan superalgebra. 
In the particular case of $\Gamma=$ Grassman algebra on $\xi_{1}, \ldots, \xi_{n}$, $\Gamma=\Gamma_{\overline{0}}+\Gamma_{\overline{1}}$, and the Poisson bracket $\{f, g\}=\sum_{i=1}^{n}(-1)^{|f|} \frac{\partial f}{\partial \xi_{i}} \frac{\partial g}{\partial \xi_{i}}$, the corresponding Jordan superalgebra $J=\Gamma+\Gamma x$ is isomorphic to the superalgebra $D_{-1}$ in the series $D_{t}$, when $t=-1$ and it is a simple Jordan superalgebra with even part $J_{\overline{0}}$ not semisimple if $n \geq 2$ (it is the one that initially did not appear in the classification by Kac).

Example. If $\Gamma=F[x]$ and $\{f(x), g(x)\}=f^{\prime}(x) g(x)-f(x) g^{\prime}(x)$, then $\Gamma+\Gamma x$ is a Jordan superalgebra. However, the previous bracket is not a Poisson bracket. This proves that it is possible to define a bracket over an associative commutative superalgebra that is not a Poisson bracket and still get a Jordan superalgebra via the Kantor process. This fact justifies the following definition:

Definition 4.1. (see [10]) A bracket $\{\cdot, \cdot\}: \Gamma \times \Gamma \rightarrow \Gamma$ is said to be a Jordan bracket if $J=\Gamma+\Gamma x$ is a Jordan superalgebra.

If the bracket is a Jordan bracket, then

(i) $D: a \mapsto\{a, 1\}$ is a derivation of $\Gamma$, and

(ii) $\{a, b c\}=\{a, b\} c+(-1)^{|a||b|} b\{a, c\}-D(a) b c$.

In particular, if $\Gamma=\left\langle a_{i}, i \in I\right\rangle$ then in order to know the action of the bracket $\{\cdot, \cdot\}$, we only need to know $D$ and $\left\{a_{i}, a_{j}\right\}$.

\section{More Examples.}

Let $\Gamma=F\left[t^{-1}, t, \xi_{1}, \ldots, \xi_{n}\right]$ be the polynomial algebra in one even Laurent variable and $n$ odd Grassmann variables. Then we can consider the following two Jordan brackets:

(a) $D=\frac{\partial}{\partial t} t, \quad\left\{\xi_{i}, \xi_{j}\right\}=-\delta_{i j}, \quad\left\{t, \xi_{i}\right\}=0$ (the Jordan bracket of Neveu-Schwarz type).

(b) $D=\frac{\partial}{\partial t}, \quad\left\{\xi_{i}, \xi_{j}\right\}=-\delta_{i j}, \quad\left\{t, \xi_{i}\right\}=0$ (the Jordan bracket of Ramond type).

King and McCrimmon characterize Jordan brackets with some relations that translate the fact that $J$ is a Jordan superalgebra. But, what are really Jordan brackets? How far are they from Poisson brackets? It can be proved that every Jordan bracket can be embedded into a Poisson bracket. Indeed, this is proved in [13].

Proposition 4.1. (a) If $\{\cdot, \cdot\}$ is a Poisson bracket on $\Gamma, u \in \Gamma_{0}$ then $\langle a, b\rangle=\{u a, u b\}$ is a Jordan bracket on $\Gamma$.

(b) All Jordan brackets can be obtained in that way. 
If $\Gamma$ has a Jordan bracket $\{\cdot, \cdot\}$, and we consider the embedding $\Gamma \hookrightarrow \Gamma\left[t^{-1}, t\right]$, we can define a new bracket on $\Gamma\left[t^{-1}, t\right]$ by

$\left\langle t^{i} a, t^{j} b\right\rangle=t^{i+j-2}\left(-(i-1) a D(b)+(-1)^{|a||b|}(j-1) b D(a)+\{a, b\}\right)$,

$a, b \in \Gamma_{0} \cup \Gamma_{1}$. This new bracket is a Poisson bracket on $\Gamma\left[t^{-1}, t\right]$ and

$$
J=J(\Gamma,\{\cdot, \cdot\}) \simeq \Gamma+t \Gamma x \leq J\left(\Gamma\left[t^{-1}, t\right],\langle,\rangle\right) .
$$

What is know about Jordan superalgebras with non-semisimple even part? Here the inspiration comes from the theory of superconformal algebras. It has been often noticed that the situation in finite dimensional algebras of prime characteristic reflects what happens in the infinite dimensional case in zero characteristic. This is the case here.

Since nothing is known about simple finite dimensional Lie superalgebras of prime characteristic, the method used by Kac to get the classification of simple finite dimensional Jordan superalgebras in characteristic 0 is not any longer available. M. Racine and E. Zelmanov, in their classification of simple finite dimensional Jordan superalgebras in prime characteristic under the assumption of semisimple even part, focus the attention on the action of the even part on the odd part. So representations and modules play a key role. Again this method does not work when we omit the assumption of semisimplicity of the even part. In this case all the ideas are inspired and follow the lines of a previous classification of some infinite dimensional Jordan superalgebras (in zero characteristic) that come associated to superconformal algebras. Let us explain these ideas.

Definition 4.2. A "superconformal algebra" is a $\mathbb{Z}$-graded, simple Lie superalgebra $L=\sum_{i \in \mathbb{Z}} L_{i}$ with $\operatorname{dim} L_{i} \leq d \forall i \in \mathbb{Z}$ and containing Virasoro.

V. Kac and van de Leur (1989) conjectured that

$$
W(1, n)=\operatorname{Der} F\left[t^{-1}, t, \xi_{1}, \ldots, \xi_{n}\right]
$$

and some of its (well known) subsuperalgebras of Cartan type give all possible examples of superconformal algebras.

Later S. J. Cheng and V. Kac found a new example of a superconformal algebra, the so called Cheng-Kac superalgebra $C K(6)$. So the conjecture was reformulated including this new example. 
Given the already mentioned relation between Jordan and Lie structuras via the Kantor-Koecher-Tits process, it seems natural to approach the problem using Jordan algebras. So we studied $\mathbb{Z}$-graded simple Jordan superalgebras, $J=\sum_{i} J_{i}$ with $\operatorname{dim} J_{i} \leq d$. Dropping the assumption $V i r \subseteq K(J)$ we have the following examples:

\section{Examples.}

1) If $\mathcal{G}$ is a finite dimensional simple Jordan superalgebra, then the loop algebra is the superalgebra $J=\mathcal{G}\left[t^{-1}, t\right]=\sum_{i \in Z} \mathcal{G} t^{i}$.

$\left.1^{\prime}\right)$ If $\mathcal{G}=\mathcal{G}_{0}+\ldots+\mathcal{G}_{n-1}$ is $\mathbb{Z} / n \mathbb{Z}$-graded, then the (twisted) loop algebra is $\mathcal{L}(G)=J=\sum_{i \in Z} \mathcal{G}_{\bar{i}} t^{i} \leq \mathcal{G}\left[t^{-1}, t\right]$.

2) Let $V=V_{\overline{0}}+V_{\overline{1}}, \quad V_{\overline{0}}=\sum_{i \in \mathbb{Z}} V_{\overline{0} i}, \quad V_{\overline{1}}=\sum_{i \in \mathbb{Z}} V_{\overline{1} i}, \operatorname{dim} V_{i} \leq$ $d$, be a $\mathbb{Z}$-graded super-vector space and $\langle\rangle:, V \times V \rightarrow F$ nondegenerate super-symmetric form. Then $J=F 1+V$ the superalgebra of the superform is another example of Jordan superalgebra of the considered type.

3) Let $V=V_{\overline{0}}+\ldots+V_{\overline{n-1}}$ be a finite dimensional $\mathbb{Z} / n \mathbb{Z}$-graded vector space, $G(V)=\sum_{i=0}^{n-1} G(V)_{\bar{i}}$ the Grassmann superalgebra, $\Gamma=\sum_{i \in Z} G(V)_{\bar{i}} t^{i}$ the (twisted) loop algebra and $\langle\rangle:, \Gamma \times \Gamma \rightarrow \Gamma$ a Jordan bracket. Then $\mathbf{J}=\boldsymbol{\Gamma}+\boldsymbol{\Gamma} \mathbf{x}$ the Kantor double superalgebra is also a $\mathbb{Z}$-graded simple Jordan superalgebra with the wanted conditions.

4) A Jordan superalgebra $J$ is of Cartan type if $J$ contains a subsuperalgebra $B$ of finite codimension such that $B^{-}+\left[B^{-}, J^{+}\right]+$ $\left[J^{-}, B^{+}\right]+B^{+}$has finite codimension in $K(J)$. (In this case $K(J)$ is a Lie superalgebra of Cartan type).

5) There exists a Jordan superalgebra $J C K(6)$ whose KantorKoecher-Tits construction gives $C K(6)$.

In a joint paper with $\mathrm{V}$. Kac and E. Zelmanov we prove that the previous examples give all possible Jordan superalgebras with the given conditions.

Theorem 4.2. [5] Let $J=\sum_{i \in Z} J_{i}$ be an infinite dimensional graded simple Jordan superalgebra in characteristic zero such that the dimensions of the graded components are uniformly bounded from above. Then $J$ is one of the algebras in 1)-5) listed above. In type 3), if $n$ is odd then there is only one bracket and $\operatorname{deg} x=0$; if $n$ is even there are two brackets, in one of them $\operatorname{deg} x=0$ (Neveu-Schwarz type) and in the other one, $\operatorname{deg} x=\frac{-n}{2}$ (Ramond type). 


\section{Cheng-Kac Jordan Superalgebras}

Cheng-Kac Jordan superalgebras appear in a more general context than the one in the above mentioned theorem. So we will explain the way in which they appear, obtaining the Cheng-Kac superalgebras in Theorem 4.2 as a particular case.

Let $Z$ be a unital associative commutative algebra and $d: Z \rightarrow Z$ a derivation. Then $C K(Z, d)=J_{\overline{0}}+J_{\overline{1}}$ is the Jordan superalgebra whose even and odd parts are free $Z$-modules of rank $4, J_{\overline{0}}=Z+$ $\sum_{i=1}^{3} w_{i} Z, J_{\overline{1}}=x Z+\sum_{i=1}^{3} x_{i} Z$ and the product is given by the following rules:

Even part

$$
w_{i} w_{j}=0, i \neq j, w_{1}^{2}=w_{2}^{2}=1, w_{3}^{2}=-1
$$

Module action of $J_{\overline{0}}$ over $J_{\overline{1}}, f, g \in Z$

\begin{tabular}{c|c|c} 
& $g$ & $w_{j} g$ \\
\hline$x f$ & $x(f g)$ & $x_{j}\left(f g^{d}\right)$ \\
\hline & & \\
\hline$x_{i} f$ & $x_{i}(f g)$ & $x_{i \times j}(f g)$
\end{tabular}

where we have used the following notation: $x_{i \times i}=0, x_{1 \times 2}=$ $-x_{2 \times 1}=x_{3}, \quad x_{1 \times 3}=-x_{3 \times 1}=x_{2}, \quad-x_{2 \times 3}=x_{3 \times 2}=x_{1}$.

Bracket on $J_{\overline{1}}$.

\begin{tabular}{c|c|c} 
& $x g$ & $x_{j} g$ \\
\hline$x f$ & $f^{d} g-f g^{d}$ & $-w_{j}(f g)$ \\
\hline$x_{i} f$ & $w_{i}(f g)$ & 0
\end{tabular}

The superalgebra $C K(Z, d)$ is simple if and only if $Z$ does not contain proper $d$-invariant ideals.

When $Z=F\left[t^{-1}, t\right]$ we get the Jordan superalgebra $J C K(6)$ that corresponds to the Cheng-Kac superconformal algebra $C K(6)$. 
Back to (unital) finite-dimensional simple Jordan.

Now we can finish the classification of simple (unital) finite dimensional Jordan superalgebras over a field $F$, $\operatorname{char} F=p>2$. Let us denote $B(m)=F\left[a_{1}, \ldots, a_{m} \mid a_{i}^{p}=0\right]$ the algebra of truncated polynomials. We will use the following result due to R. Block (1969):

A finite dimensional algebra $A$ is differentially simple if and only if either $A$ is simple or char $F=p$ and $A \simeq \mathcal{G} \otimes B(m)$ with $\mathcal{G}$ a simple algebra.

Denoting $B(m, n)=B(m) \otimes G(n)$, where $G(n)=\left\langle 1, \xi_{1}, \ldots, \xi_{n}\right\rangle$, the following result was proved jointly with Zelmanov:

Theorem 4.3. [14] Let $J=J_{\overline{0}}+J_{\overline{1}}$ be a finite dimensional simple unital Jordan superalgebra over an algebraically closed field $F$, char $F=p>2, J_{\overline{0}}$ not semisimple. Then $J$ is a Kantor double of a superalgebra $B(m, n): \mathbf{J}=\mathbf{B}(\mathbf{m}, \mathbf{n})+\mathbf{B}(\mathbf{m}, \mathbf{n}) \mathbf{x}$ or $J$ is a ChengKac superalgebra: $\mathbf{J} \simeq \mathbf{C K}(\mathbf{B}(\mathbf{m}), \mathbf{d})$.

\section{Nonunital Jordan Superalgebras}

We have already seen some examples of nonunital Jordan superalgebras: the 3-dimensional Kaplansky superalgebra $K_{3}$ and the 9dimensional Kac superalgebra $K_{9}$ in characteristic 3. Another example can be constructed in the following way:

Let $Z$ be an associative, commutative $F$-algebra and $D: Z \longrightarrow Z$ a derivation such that:

(i) $Z$ has no nonzero proper $D$-invariant ideals,

(ii) The only constants of the derivation $D$ in $Z$ are scalars $\alpha 1$, $\alpha \in F$.

Then $V_{\frac{1}{2}}(Z, D)=J_{0}+J_{1} \quad$ with $J_{0}=Z, J_{1}=Z$, the product in $J_{0}$ equal to the product in $Z, a \cdot x=\frac{1}{2} a x$ for elements $a \in J_{0}, x \in J_{1}$, (juxtaposition denotes the product in $Z$ ) and $[x, y]=(x D) y-(y D) x$, $x, y \in J_{\overline{1}}$, is a non-unital Jordan superalgebra.

Zelmanov, in a paper to appear in the Proceedings of a Conference in Taiwan, 2000, proved that the three examples above give all possible simple non-unital Jordan superalgebras.

Theorem 5.1. Let $J$ be a finite dimensional simple nonunital Jordan superalgebra over a field $F$. Then $J$ is isomorphic to one of the following superalgebras:

1. The Kaplansky superalgebra, 
2. The degenerate Kac superalgebra $K_{9}(F), \operatorname{char} F=3$,

3. A superalgebra $V_{\frac{1}{2}}(Z, D)$.

In the same paper, the structure of semisimple Jordan superalgebras (that is, without nonzero nilpotent ideals) is given by Zelmanov.

Theorem 5.2. Let $J$ be a finite dimensional Jordan superalgebra over $F$, char $F \neq 2$. Then $J$ is semi-simple if and only if $J$ is a direct sum of simple Jordan superalgebras and unital hulls

$$
H_{K}\left(J_{1} \oplus \cdots \oplus J_{r}\right)=J_{1} \oplus \cdots \oplus J_{r} \oplus K 1
$$

with $K / F$ an extension and $J_{1}, \ldots, J_{r}$ simple nonunital Jordan superalgebras.

$$
J \simeq \bigoplus_{i=1}^{s}\left(J_{i 1} \oplus \cdots \oplus J_{i r_{i}} \oplus K_{i} 1\right) \oplus J_{1} \oplus \cdots \oplus J_{r}
$$

with $J_{1}, \cdots, J_{r}$ simple Jordan superalgebras, $K_{1}, \cdots, K_{s}$ fields extensions of $F$ and for $i$ fixed in $\{1,2, \ldots, s\}, J_{i 1}, \ldots, J_{i r_{i}}$ are simple non-unital Jordan superalgebras over the field $K_{i}$.

\section{Speciality}

Now we will try to review what is known about the speciality of all Jordan superalgebras that have appeared in the different classification theorems

- The Kantor Double of a bracket of vector field type $(\{a, b\}=$ $a^{\prime} b-a b^{\prime}$, where' is a derivation) is special. (McCrimmon, 1992)

- The Kantor Double of $\{f, g\}=\frac{\partial f}{\partial x} \frac{\partial g}{\partial y}-\frac{\partial f}{\partial y} \frac{\partial g}{\partial x}$ on $F[x, y]$ is exceptional. (See [15].)

Shestakov (1993) proved that a Kantor Double of a Poisson bracket $\langle\cdot, \cdot\rangle: \Gamma \times \Gamma \rightarrow \Gamma$ is special if and only if $\langle\langle\Gamma, \Gamma\rangle, \Gamma\rangle=(0)$.

He also proved that a Kantor Double of a Poisson bracket is ispecial (that is, homomorphic image of a special superalgebra). The main idea here is to prove that if the bracket is quantizable, then the corresponding Jordan superalgebra is special. Of course not every bracket is quantizable, but the free Poisson bracket is such and every Kantor double is an epimorphic image of a Kantor double of a free Poisson bracket, so it is i-special. 
What about Jordan brackets? Using the above mentioned result by Shestakov and our joined result with Shestakov and Zelmanov proving that every Jordan bracket is embeddable into a Poisson bracket, we conclude that a Kantor Double of a Jordan bracket is i-special.

There is no characterization of speciality of Jordan brackets as nice as the one given by Shestakov for Poisson brackets. However some (important) particular cases are known. These cases cover all situations that appear in the classification theorems, with the exception of Cheng-Kac superalgebras.

Let us assume that $J=\Gamma+\Gamma x$ does not contain nonzero nilpotent ideals. Then:

- If $\Gamma=\Gamma_{\overline{0}}$ then $J$ is special if and only if $\langle$,$\rangle is of vector field type.$ - If $\Gamma_{\overline{1}} \Gamma_{\overline{1}} \neq(0)$ (which means in the superalgebras appearing in the classification theorem that there are at least 2 Grassmann variables), then $J$ is exceptional.

- If $\Gamma=\Gamma_{\overline{0}}+\Gamma_{\overline{0}} \xi_{1}$, with $\left\langle\Gamma_{\overline{0}}, \xi_{1}\right\rangle=(0),\left\langle\xi_{1}, \xi_{1}\right\rangle=-1$ then $J$ is special if and only if $\langle\rangle:, \Gamma_{\overline{0}} \times \Gamma_{\overline{0}} \rightarrow \Gamma_{\overline{0}}$ is of vector field type.

Only Cheng-Kac superalgebras are not covered by the previous result. Contrary to what could be initially thought, these algebras turned out to be special.

Theorem 6.1. [13] The Cheng-Kac superalgebras $C K(Z, d)$ are special.

The embedding extends McCrimmon's embedding for vector field type brackets.

Let $W=\langle R(a), a \in Z, d\rangle$ be the algebra of differential operators on $Z$, that is, the algebra generated by the derivation $d$ and right multiplications by elements of $Z$ and let's consider $R=R_{\overline{0}}+R_{\overline{1}}=$ $\mathcal{M}_{4 \times 4}(W)$ the superalgebra whose even and odd part are respectively

$$
R_{\overline{0}}=\left(\begin{array}{cccc}
W & 0 & W & 0 \\
0 & W & 0 & W \\
W & 0 & W & 0 \\
0 & W & 0 & W
\end{array}\right), R_{\overline{1}}=\left(\begin{array}{cccc}
0 & W & 0 & W \\
W & 0 & W & 0 \\
0 & W & 0 & W \\
W & 0 & W & 0
\end{array}\right)
$$

and the embedding $\varphi: C K(Z, d) \rightarrow R=\mathcal{M}_{4}(W)$, given by: 


$$
\begin{gathered}
\varphi(a)=\left(\begin{array}{llll}
a & 0 & 0 & 0 \\
0 & a & 0 & 0 \\
0 & 0 & a & 0 \\
0 & 0 & 0 & a
\end{array}\right), a \in Z, \varphi\left(w_{1}\right)=\left(\begin{array}{cccc}
-1 & 0 & 0 & 0 \\
0 & -1 & 0 & 0 \\
0 & 0 & -1 & 0 \\
0 & 0 & 0 & -1
\end{array}\right), \\
\varphi\left(w_{2}\right)=\left(\begin{array}{llll}
0 & 0 & 1 & 0 \\
0 & 0 & 0 & 1 \\
1 & 0 & 0 & 0 \\
0 & 1 & 0 & 0
\end{array}\right), \varphi\left(w_{3}\right)=\left(\begin{array}{cccc}
0 & 0 & -1 & 0 \\
0 & 0 & 0 & 1 \\
1 & 0 & 0 & 0 \\
0 & -1 & 0 & 0
\end{array}\right), \\
\varphi(x)=\left(\begin{array}{cccc}
0 & 0 & 0 & 2 d \\
0 & 0 & -1 & 0 \\
0 & -2 d & 0 & 0 \\
1 & 0 & 0 & 0
\end{array}\right), \varphi\left(x_{1}\right)=\left(\begin{array}{cccc}
0 & 0 & 0 & -1 \\
0 & 0 & 0 & 0 \\
0 & -1 & 0 & 0 \\
0 & 0 & 0 & 0
\end{array}\right), \\
\varphi\left(x_{2}\right)=\left(\begin{array}{cccc}
0 & -1 & 0 & 0 \\
0 & 0 & 0 & 0 \\
0 & 0 & 0 & 1 \\
0 & 0 & 0 & 0
\end{array}\right), \varphi\left(x_{3}\right)=\left(\begin{array}{cccc}
0 & 1 & 0 & 0 \\
0 & 0 & 0 & 0 \\
0 & 0 & 0 & 1 \\
0 & 0 & 0 & 0
\end{array}\right) .
\end{gathered}
$$

This embedding also gives a new representation of the Lie ChengKac superalgebra $C K(6)$ as $8 \times 8$ matrices over $W=\left\langle t^{-1}, t, \partial_{t}\right\rangle$. This provides a new proof that $C K(6)$ is a Lie superalgebra. Also a new proof of the fact that $C K(Z, d)$ is a Jordan superalgebra follows immediately.

\section{More Relations with Lie Superalgebras}

Tits constructed a Lie algebra from an alternative trace algebra $A$ with a trace function $t: A \rightarrow F$, that is commutative, $t(a b)=t(b a)$, and associative $t((a b) c)=t(a(b c))$, and satisfies a generic HamiltonCayley trace identity of degree $2: x^{2}-2 t(x) x+\left(2 t(x)^{2}-t\left(x^{2}\right)\right) 1$, and a Jordan algebra $J$ with a similar trace function, $t: J \rightarrow F$ that satisfies a Cayley-Hamilton identity of degree $3: x^{3}-3 t(x) x^{2}+$ $\left(\frac{9}{2} t(x)^{2}-\frac{3}{2} t\left(x^{2}\right)\right) x-\left(t\left(x^{3}\right)-\frac{9}{2} t\left(x^{2}\right) t(x)+\frac{9}{2} t(x)^{3}\right) 1$.

The algebra constructed by Tits is $T(A, J)=\mathcal{D}(A) \oplus\left(A_{0} \otimes J_{0}\right) \oplus$ $\mathcal{D}(J)$, where $\mathcal{D}(A)$ (resp. $\mathcal{D}(J))$ is a derivation algebra of $A$ (resp. $J$ ) containing the inner derivations and $A_{0}$ and $J_{0}$ denote the sets of elements of $A$ and $J$ respectively of zero trace. The structure of a Lie algebra on $\mathcal{L}=T(A, J)$ is defined in such a way that $\mathcal{D}(A) \oplus \mathcal{D}(J)$ is 
a subalgebra with the usual product and $A_{0} \otimes J_{0}$ is a $\mathcal{D}(A) \oplus \mathcal{D}(J)$ module in the usual way $([a \otimes x, D+E]=a D \otimes x+a \otimes x E)$.

The most interesting cases are obtained when $A$ is a composition algebra and $J$ is either $F$ or a simple central Jordan algebra of degree 3.

The situation is reflected in the so called Magic Freudenthal-Tits Square

\begin{tabular}{c|c|c|c|c|c|c|c} 
& $F$ & $H_{3}(F, \gamma)$ & $B^{+}$or $H(B)$ & $H\left(Q_{3}, \gamma\right)$ & $H_{3}(\mathcal{O})$ & $J_{3}$ & $J_{4}$ \\
\hline$F$ & 0 & $A_{1}$ & $A_{2}$ & $C_{3}$ & $F_{4}$ & & \\
\hline$F[u]$ & 0 & $A_{2}$ & $A_{2} \oplus A_{2}$ & $A_{5}$ & $E_{6}$ & & \\
\hline $\mathcal{Q}$ & $A_{1}$ & $C_{3}$ & $A_{5}$ & $D_{6}$ & $E_{7}$ & & \\
\hline $\mathcal{O}$ & $G_{2}$ & $F_{4}$ & $E_{6}$ & $E_{7}$ & $E_{8}$ & $G(3)$ & $F(4)$
\end{tabular}

Notice that all exceptional Lie algebras appear in the last row of this square.

Benkart and Zelmanov [1] proved that the previous magic square can be extended to superalgebras. Indeed, considering still the alternative algebra of octonions, but the Jordan superalgebras $J_{3}$ (the unital 3-dimensional Jordan superalgebra of a superform) and $J_{4}$ (the Jordan superalgebra in the family $D_{s}$ for $s=2$ ), with some specific trace functions and using the Tits construction, the (31dimensional) Lie superalgebra $G(3)$ and the (40-dimensional) Lie superalgebra $F(4)=K\left(K_{10}\right)$ can be obtained.

\section{REFERENCES}

[1] G. Benkart and E. Zelmanov, Lie algebras graded by finite root systems and intersection matrix algebras, Invent. Math. 126 (1996), 1-45.

[2] S. J. Cheng and V. G. Kac, A new $N=6$ superconformal algebra, Comm. Math. Phys. 186 (1997).

[3] V. G. Kac, Lie superalgebras, Adv. in Math. 26 (1977), 8-96.

[4] V. G. Kac, Classification of simple $Z$-graded Lie superalgebras and simple Jordan superalgebras, Comm. Algebra 5 (1977), 1375-1400.

[5] V. G. Kac, C. Martínez and E. Zelmanov, Graded simple Jordan superalgebras of growth one, Mem. Amer. Math. Soc. 150 (2001).

[6] I. L. Kantor, Connections between Poisson brackets and Jordan and Lie superalgebras, Lie theory, differential equations and representation theory, Montreal (1989), 213-225. 
[7] I. L. Kantor, Jordan and Lie superalgebras defined by Poisson brackets, Algebra and Analisis, 55-79, 1989; Amer. Math. Soc. Transl. Ser. (2), 151 (1992).

[8] I. Kaplansky, Superalgebras, Pacific J. of Math. 86 (1980), 93-98.

[9] I. Kaplansky, Graded Jordan algebras I, preprint.

[10] D. King and K. McCrimmon, The Kantor construction of Jordan superalgebras, Comm. Algebra 20 (1992), 109-126.

[11] D. King and K. McCrimmon, The Kantor doubling process revisited, Comm. Algebra 23 (1995), 357-372.

[12] S. V. Krutelievich, Simple Jordan superpairs, Comm. Algebra 25 (1977), $2635-2657$.

[13] C. Martínez, I. Shestakov and E. Zelmanov, Jordan algebras defined by brackets, J. London Math. Soc.(2) 64 (2001),357-368.

[14] C. Martínez and E. Zelmanov, Simple finite dimensional Jordan superalgebras of prime characteristic, J. Algebra 236 (2001), 575-629.

[15] K. McCrimmon, Speciality and nonspeciality of two Jordan superalgebras, J. Algebra 149 (1992), 326-351.

[16] K. McCrimmon, Kaplansky superalgebras, J. Algebra 164 (1994), 656-694.

[17] K. McCrimmon, Jordan supersystems, Nova Sci. Publish., Commack, NY, 1992, 17-36.

[18] Y. Medvedev and E. Zelmanov, Some counterexamples in the theory of Jordan algebras, S. González and H.C. Myung (eds.), Nonassociative Algebraic Models, Nova Sci. Publish., Commack, NY, 1992, 1-16.

[19] N. A. Pisarenko, The Wedderburn decomposition in finite dimensional alternative superalgebras, Algebra and Logic 32 (1993), 231-238.

[20] N. A. Pisarenko, The structure of alternative superbimodules, Algebra and Logic 33 (1994) 386-397.

[21] M. Racine, Primitive superalgebras with superinvolution, J. Algebra 206 (1998) 588-614.

[22] M. Racine and E. Zelmanov, Simple Jordan superalgebras, S. González (ed.), Nonassociative Algebra and its Applications, Kluwer Acad. Publish., 1994, 344-349.

[23] M. Racine and E. Zelmanov, Classification of simple Jordan superalgebras with semisimple even part, to appear in J. Algebra.

[24] I. Shestakov, Superalgebras and counterexamples, Siberian Math. J. 32 (1991), 1052-1060.

[25] I. Shestakov, Superalgebras as a building material for constructing counterexamples, Nova Sci. Publish., Commack, NY, 1992, 53-64.

[26] I. Shestakov, Quantization of Poisson superalgebras and speciality of Jordan Poisson superalgebras, Algebra and Logic 32 (1993), 309-317.

[27] I. Shestakov, Quantization of Poisson superalgebras and speciality of Jordan Poisson superalgebras, S. González (ed.), Nonassociative Algebra and its Applications, Kluwer Acad. Publish. 1994, 372-378.

[28] I. Shestakov, Quantization of Poisson algebras and weak speciality of related Jordan superalgebras, Dokl. Akad. Nauk 334 (1994), 29-31.

[29] I. Shestakov, Prime alternative superalgebras of arbitrary characteristic, Algebra and Logic 36 (1997), 389-412. 
[30] I. Shestakov, Alternative and Jordan superalgebras, Siberian Advances in Mathematics 9 (1999), 83-99.

[31] I. Shestakov and E. Zelmanov, Prime alternative superalgebras and the nilpotency of the radical of a free alternative algebra, Izv. Akad. Nauk. SSSR 54 (1990), 676-693.

[32] A. S. Shtern, Representation of an exceptional Jordan superalgebra, Funktsional Anal. i Prilozhen 21 (1987), 93-94.

[33] A. S. Shtern, Representations of finite dimensional Jordan superalgebras of Poisson brackets, Comm. Algebra 23 (1995), 1815-1823.

[34] V. G. Skosyrskii, Prime Jordan algebras and the Kantor construction, Algebra and Logic 33 (1994), 169-179.

[35] C. T. C. Wall, Graded Brauer groups, J. Reine Angew Math. 213 (1964), 187-199.

[36] E. Zelmanov, Semisimple finite dimensional Jordan superalgebras, Y. Fong, A. A. Mikhalev, E. Zelmanov (eds.), Lie Algebras, Rings and Related Topics, Springer-Verlag, Hong Kong 2000, 227-243.

[37] E. Zelmanov, Special Jordan nil-algebras of bounded index, Algebra and Logic 22 (1983), 626-635.

Consuelo Martínez,

Departamento de Matemáticas,

Universidad de Oviedo,

C/ Calvo Sotelo, s/n,

33007 Oviedo, Spain,

chelo@pinon.ccu.uniovi.es

Received on 19 May 2001 and in revised form on 21 May 2003. 